\section{Lubricin is expressed in chondrocytes derived from osteoarthritic cartilage encapsulated in poly (ethylene glycol) diacrylate scaffold}

\author{
G. Musumeci, ${ }^{1}$ C. Loreto, ${ }^{1}$ M.L. Carnazza, ${ }^{1}$ \\ F. Coppolino, ${ }^{2}$ V. Cardile, ${ }^{3}$ R. Leonardi ${ }^{4}$ \\ 'Department of Bio-Medical Sciences, \\ Human Anatomy section, University of \\ Catania; ${ }^{2}$ Department of Bio-Medical \\ Sciences, Phisiology section, University \\ of Catania; ${ }^{3}$ Radiology, University of \\ Palermo; ${ }^{4}$ Department of Medical and \\ Surgical Sciences, II Dental Unity, \\ University of Catania, Italy
}

\section{Abstract}

Osteoarthritis $(\mathrm{OA})$ is characterized by degenerative changes within joints that involved quantitative and/or qualitative alterations of cartilage and synovial fluid lubricin, a mucinous glycoprotein secreted by synovial fibroblasts and chondrocytes. Modern therapeutic methods, including tissue-engineering techniques, have been used to treat mechanical damage of the articular cartilage but to date there is no specific and effective treat ment. This study aimed at investigating lubricin immunohistochemical expression in cartilage explant from normal and $\mathrm{OA}$ patients and in cartilage constructions formed by Poly (ethylene glycol) (PEG) based hydrogels (PEG-DA) encapsulated $\mathrm{OA}$ chondrocytes. The expression levels of lubricin were studied by immunohistochemistry: i) in tissue explanted from $\mathrm{OA}$ and normal human cartilage; ii) in chondrocytes encapsulated in hydrogel PEGDA from OA and normal human cartilage. Moreover, immunocytochemical and western blot analysis were performed in monolayer cells from $\mathrm{OA}$ and normal cartilage. The results showed an increased expression of lubricin in explanted tissue and in monolayer cells from normal cartilage, and a decreased expression of lubricin in $\mathrm{OA}$ cartilage. The chondrocytes from OA cartilage after 5 weeks of culture in hydrogels (PEGDA) showed an increased expression of lubricin compared with the control cartilage. The present study demonstrated that $\mathrm{OA}$ chondrocytes encapsulated in PEGDA, grown in the scaffold and were able to restore lubricin biosynthesis. Thus our results suggest the possibility of applying autologous cell transplantation in conjunction with scaffold materials for repairing cartilage lesions in patients with $\mathrm{OA}$ to reduce at least the progression of the disease.

\section{Introduction}

Articular cartilage homeostasis is the result of an intricate interplay between anabolic and catabolic, anti- and pro-inflammatory, antiand pro-apoptotic mediators. These processes act also as protective mechanisms whether there is a sub lethal injury characterized by an increased survival of chondrocytes, which play, in that way, a central role in the growth of the new cartilage and extracellular matrix. ${ }^{1,2}$ As chondrocytes represent versatile regulators for this cartilage equilibrium, they are highly sensitive and responsive to mechanical, biochemical and other stressful stimuli. ${ }^{3}$ However, any kind of cartilage injury can lead to an irreversible cartilage loss, since differentiated chondrocytes do not divide and hence do not compensate for these defects. Additionally, cartilage homeostasis is disturbed by the predominance of directly and indirectly catabolic acting factors such as pro-inflammatory cytokines, which can result in cartilage damage and secondary osteoarthritis (OA). ${ }^{4}$

Osteoarthritis, also known as degenerative arthritis, is a multifactorial degenerative joint disease, characterized by degenerative changes within joints that involved quantitative and/or qualitative alterations of cartilage and synovial fluid lubricin, a mucinous glycoprotein secreted by synovial fibroblasts and chondrocytes. It can be classified into either primary or secondary depending on whether there is 0 an identifiable underlying cause or not. The progressive erosion of articular cartilage, leading to joint space narrowing, subchondral sclerosis, subchondral cyst, synovial inflammation, and marginal osteophyte formation, characterizes the disease process of $0 \mathrm{~A}^{5-7}$ It is known that $\mathrm{OA}$ is the result of mechanical and biological processes that modify cartilage homeostasis. ${ }^{8,9}$

Modern therapeutic methods, including tissue-engineering techniques, have been used to treat the mechanical damage of articular cartilage. Among a number of these recently developed methods, the autologous chondrocyte transplantation (ACT) seems promising. In this procedure, the chondrocytes were isolated from a cartilage, these chondrocytes are cultured in vitro, and subsequently, they can be transplanted into the damaged area. Some studies have revealed that these cells are capable of producing, in vivo, an extracellular matrix of chemical composition and biomechanical properties similar to those of normal hyaline cartilage. ${ }^{10-12}$ In this respect, chondrocytes of the middle and deep zones synthesize aggrecan at high rates, and thereby contribute to the ability of cartilage to bear compressive loads; on the other hand, the chondrocytes of the superficial zone exhibit a number of spe-
Correspondence: Giuseppe Musumeci, Department of Bio-Medical Sciences, Human Anatomy section, University of Catania, via S. Sofia 87, 95123 Catania, Italy.

Tel. +39.095.3782043 - Fax: +39.095.3782034.

E-mail: g.musumeci@unict.it

Key words: osteoarthritis, hydrogels (PEG-DA) chondrocytes, lubricin.

Acknowledgments: this study was supported by grants provided by the Medicine and Surgery Faculty, University of Catania. The authors would like to thank Dr. Corrado Loreto from Orthopaedic and Traumatology Unit, Hospital of Suzzara, Mantova, Italy, for providing surgical materials.

Contributions: GM, study conception and design, data integrity and data analysis responsibility; CL, RL, FC, data acquisition; CL, RL, VC, data analysis and interpretation; GM, CL, MLC, article drafting. All authors were involved in drafting the article or revising it critically for important intellectual content and approved the final version.

Received for publication: 4 July 2011. Accepted for publication: 31 July 2011.

This work is licensed under a Creative Commons Attribution NonCommercial 3.0 License (CC BYNC 3.0).

(C) Copyright G. Musumeci et al., 2011 Licensee PAGEPress, Italy

European Journal of Histochemistry 2011; 55:e31 doi:10.4081/ejh.2011.e31

cific functions including the synthesis and secretion of superficial zone protein (SZP) also known as proteoglycan 4 (Prg4) or lubricin.

Lubricin $(227.5-\mathrm{kDa})$ is a mucinous glycoprotein secreted by synovial fibroblasts and chondrocytes. ${ }^{13}$ This molecule is partly responsible for the lubrication of apposed and pressurized cartilage, surfaces enabling low friction levels. ${ }^{14-16}$ Lubricin provides essential chondroprotective properties to articular cartilage as shown by the lack of cartilage surface integrity and surface disruption in lubricin knockout mice. ${ }^{17}$ Furthermore, quantitative and/or qualitative alterations of cartilage and synovial fluid lubricin have been described in OA. Lubricin has been localized on the surface of multiple synovial tissues, including cartilage, meniscus, ligament, and tendon, ${ }^{18-24}$ whereupon it acts as a boundary lubricant and as a deterrent against abnormal protein deposition and/or cellular adhesion. In addition, lubricin contributes to the load dissipating elasticity of synovial fluid. Thus, the expression of lubricin in chondrocytes delivered on absorbable scaffolds seems to be absolutely neccessary. As regards this, some studies have demonstrated that chondrocytes from the 
superficial zone of bovine cartilage, used in explants, monolayer, transplant and alginate constructions are able to express lubricin..$^{25,26}$

However, to the knowledge of authors, no investigation has ever dealt with lubricin expression in human autologus chondrocytes derived from normal and $\mathrm{OA}$ cartilage and encapsulated into scaffold. Accordingly, this study aimed at investigating lubricin immunohistochemical, immunocytochemical expression and western blot analysis in cartilage explant from normal and $\mathrm{OA}$ patients and in cartilage construction formed by Poly (ethylene glycol) (PEG) based hydrogels (PEG-DA) encapsulated $\mathrm{OA}$ chondrocytes.

\section{Materials and Methods}

\section{Subjects}

Osteoarthritic cartilage was obtained from 25 patients, 15 males and 10 females, with similar height and weight, who underwent knee joint replacement at the Orthopaedic and Traumatology Unit, Hospital of Suzzara, Mantova, Italy, under an approved Institutional Review Board protocol after obtaining the required informed consent from the patients. Surgery was performed because of the pain and the functional impairment. The median of the age of the OA patients was 56 (range 34-78 years). Tissue samples were graded according to the histopathological Mankin scores ${ }^{27}$ and included only pieces of moderate-to-severe $\mathrm{OA}$ (Mankin score 6-14). The patients were not smokers, occasionally taking NSAIDs (nonsteroidal anti-inflammatory drugs) in addition to the classical treatment for knee OA composed of hot packs, therapeutic ultrasound and terminal isometric exercises. The females did not take estrogen replacement therapy (ERT) that may influences the physiological homeostasis of the joint.

The diagnosis was based on clinical and radiological criteria, included physical examination, X-ray imaging and MRI of the injured knee. According to Ahlbäck classification (grade IV, moderate bone attrition) and Kellgren \& Lawrence classification patients had an OA of the knee of grade 3 or 4 with moderate diminution of joint space or joint space greatly impaired with sclerosis of subchondral bone. ${ }^{28}$ Samples used as the control were obtained from 16 patients with similar height and weight to patients with $0 A$ knee, 8 males and 8 females, without history of primary or secondary arthritis, from the same Hospital, under an approved Institutional Review Board protocol and after the informed consent obtained from the patients. The median of the age of these patients was 40 (range 35-59 years). Knee joint were removed because of traumatic events. Macroscopic and microscopic examination of the knee showed no signs of degenerative or inflammatory joint disease.

\section{Isolation of chondrocytes and culture conditions}

To isolate the chondrocytes from $\mathrm{OA}$ and non $\mathrm{OA}$ articular cartilage, they were incubated in Dulbeco's modified eagle's medium (DMEM, GIBC0, Grand Island, NY, USA) containing $0.2 \%$ collagenase (Worthington Biochemical Corporation, Lakewood, NJ, USA) and 5\% FBS (fetal bovine serum, GIBC0) for $14-16 \mathrm{~h}$ at $37^{\circ} \mathrm{C}$ and $5 \% \mathrm{CO}_{2}$. The resulting cell suspension was then filtered through $70 \mu \mathrm{m}$ nylon filters (Cell Strainer; Falcon, Franklin Lakes, NJ, USA) and washed three times with phosphate buffered saline (PBS) containing $100 \mathrm{U} / \mathrm{ml}$ penicillin and $100 \mu \mathrm{g} / \mathrm{mL}$ streptomycin. The number and size destribution of the isolated cells were then determined with a Z2 Coulter Counter and Size Analyzer (Beckman Coulter, Inc., Palo Alto, CA, USA). After isolation, the chondrocytes were plated onto separate $10 \mathrm{~cm}$ tissue culture dishes at a density of 10,000 cells $/ \mathrm{cm}^{2}$. Cells were incubated at $37^{\circ} \mathrm{C}$ and $5 \% \mathrm{CO}_{2}$ in chondrocyte medium composed of DMEM containing 10\% fetal bovine serum, $0.4 \mathrm{mM}$ proline, $50 \mu \mathrm{g} / \mathrm{mL}$ ascorbic acid, $10 \mathrm{mM}$ HEPES, $0.1 \mathrm{mM}$ non-essential amino acid, and $100 \mathrm{U} / \mathrm{mL}$ penicillin and 100 $\mu \mathrm{g} / \mathrm{mL}$ streptomycin. Culture medium was changed twice weekly. The cells were observed with an Axioplan Zeiss light microscope (Germany) and photographed with a digital camera (Canon, Japan).

\section{Cell encapsulation in hydrogels (PEGDA)}

Cells were encapsulated in PEGDA hydrogels for 3, 4 and 5 weeks. Briefly, chondrocytes (passage 3 or 4 ) were suspended in $10 \%(\mathrm{w} / \mathrm{v})$ PEGDA (SunBio, Orinda, CA, USA) solution in sterile PBS with $100 \mathrm{U} / \mathrm{mL}$ penicillin and 100 $\mu \mathrm{g} / \mathrm{mL}$ streptomycin (Gibco). The photoinitiator Irgacure 2959 (Ciba Specialty Chemicals, Tarrytown, NY, USA) was used at $0.05 \%(\mathrm{w} / \mathrm{v})$ final concentration. The photo initiation and encapsulation processes have been previously determined to be biocompatible. Chondrocytes were resuspended in $95 \mu \mathrm{L}$ of polymer solution at a concentration of $20 \times 10^{6}$ cells per milliliter. The cell-polymer solution was added to a cylindrical silicone-tubing mold with an internal diameter of $4.75 \mathrm{~mm}$ and a construct height of $5 \mathrm{~mm}$. The tubing was attached to the glass microscope slide using silicon lubricant. Prepolymer (macromer) was exposed to UV light $(365 \mathrm{~nm})$ for $5 \mathrm{~min}$ to induce gelling. Constructions were removed from the molds and cultured at $37^{\circ} \mathrm{C}, 5 \%$ carbon dioxide in chondrocyte medium. The culture medium was changed twice weekly. The scaffolds were photographed with a digital camera (Canon, Tokio, Japan).

\section{Fixation, dehydration and embed- ding of hydrogels}

The hydrogel was fixed by incubating it in $500 \mu \mathrm{L}$ of $4 \%$ paraformaldehyde (w/v) in PBS (Invitrogen), pH 7.4 overnight ( 12 h). Several steps involving temperature and $\mathrm{pH}$ changes were necessary to dissolve this chemical (to dissolve the paraformaldehyde, heat the solution to $55^{\circ} \mathrm{C}$ while stirring. Once dissolved, $1 \mathrm{~N} \mathrm{NaOH}$ drop by drop was added to the solution until it became clear. Dilute $\mathrm{HCl}$ was use to lower the $\mathrm{pH}$ to 7.4). Then the hydrogel was dehydrated using the following sequence: $70 \%$ EtOH x 1 h, $80 \%$ EtOH x 1.5 h, 95\% EtOH x 12 h, $100 \%$ EtOH x 1.5 h-repeat twice, Xylene $\mathrm{x} 1 \mathrm{~h}$.

The hydrogel was placed in a metal mold (and embed in paraffin at $60^{\circ} \mathrm{C}$ overnight $(\sim 12$ h). The paraffin was poured out and replaced with fresh paraffin. An embedding cassette (Fisher) was placed on top of the mold and additional paraffin was added to fill the cassette. Cooled for $1 \mathrm{~h}$ using the cold surface of the embedding station (the embedding system used was Leica EG1150). Sections of 4-5 $\mu \mathrm{m}$ thick were cut from paraffin blocks using a microtome and they were mounted on salinecoated slides and stored at room temperature.

\section{Immunohistochemistry}

For immunohistochemical analysis, sections were incubated for $30 \mathrm{~min}$ in $0.3 \%$ $\mathrm{H}_{2} \mathrm{O}_{2}$ /methanol to quench endogenous peroxidase activity then rinsed for 20 min with phosphate-buffered saline (PBS; Bio-Optica, Milano, Italy). The sections were irradiated (5 min $x 3$ ) in capped polypropylene slide-holders with citrate buffer ( $\mathrm{pH} \mathrm{6)}$, using a microwave oven $(750 \mathrm{~W})$ to unmask antigenic sites. Then, the sections were incubated with diluted rabbit polyclonal anti-lubricin antibody (abcam, Cambridge Science Park, Cambridge, CB4 0FL, UK), (diluted 1:50 in PBS) overnight at $4^{\circ} \mathrm{C}$. The secondary antibody, biotinylated mouse/anti-rabbit IgG was applied (for $30 \mathrm{~min}$ at RT), followed by the avidin-biotin-peroxidase complex (Vector Elite Kit Abbott, Chicago, IL, USA) for $30 \mathrm{~min}$, at room temperature. The immunoreaction was visualized by incubating the sections for $4 \mathrm{~min}$ in a $0.1 \% 3,3^{\prime}$ diaminobenzidine and $0.02 \%$ hydrogen peroxide solution (DAB substrate kit, Vector Laboratories, CA, USA). The sections were lightly counterstained with Mayer's haematoxylin (Histolab Products AB, Goteborg, Sweden) mounted in GVA mount (Zymed, Laboratories Inc., San Francisco, CA, USA) 
observed with an Axioplan Zeiss light microscope (Germany) and photographed with a digital camera (Canon, Japan).

\section{Immunocytochemistry}

Immunostaining for lubricin was performed on control and $\mathrm{OA}$ cell cultures in cells after fourth passages in monolayer cultured. Briefly, cells were fixed with $4 \%$ phosphate-buffered paraformaldehyde, for $20 \mathrm{~min}$ at room temperature; quenching was performed with a solution of $2 \%$ hydrogen peroxide $\left(\mathrm{H}_{2} \mathrm{O}_{2}\right)$ and $10 \%$ methanol in phosphate-buffer saline (PBS) for $1 \mathrm{~min}$. To permeabilize the cells, fresh $0.3 \%$ Triton X-100 solutions in PBS was used for 5 min. The cells were then treated with $5 \%$ bovine serum albumin (BSA) in PBS for 1 hour at room temperature. BSA is a blocking agent used to prevent non-specific binding of the antibody. After blocking, the cells were incubated overnight with a primary Rabbit antiPRG4 unconjugated (1:200 dilution, Millipore, Billerica, MA, USA) antibody. A fluoresceine isothiocyanate (FITC) labeled anti-rabbit antibody (1:100, Santa Cruz Biotechnology, Inc. Santa Cruz, CA, USA) was used as a secondary antibody. Cover slips containing the cells were washed and mounted in PBS/glycerol (50:50) and placed on glass microscope slides. In all instances negative controls without primary antibody were performed. Cover slips were analyzed using a Nikon Eclipse TE200 fluorescence microscope and photographed with a digital camera (Canon) and positive cells were counted over the entire cover slips.

\section{Evaluation of immunohistochem- istry and immunocytochemistry}

The lubricin-staining status was identified as either negative or positive. Immunohistochemistry positive staining was defined as the presence of a brown detection chromogen on the edge of the hematoxylin-stained cell nucleus, distributed within the cytoplasm or in the immediate lacunar/pericellular space evaluated by the light microscope. Immunocyto chemistry positive staining was defined as the presence of fluorescence detection on the edge of the black background, distributed within the cytoplasm or in the immediate lacunar/pericellular space evaluated by the fluorescence microscope.

Stain intensity and the proportion of immunopositive cells were assessed also by light and fluorescence microscopy. Intensity of staining (IS) was graded on a scale of $0-4$, according to the following assessment: 0 , no detectable staining; 1 , weak staining; 2 , moderate staining; 3 , strong staining; 4 , very strong staining. The percentage of Lubricin-1 immunopositive cells (Extent Score=ES) was independently evaluated by 3 investigators ( 2 anatomical morphologists and one histologist) and scored as a percentage of the final number of 100 cells in five categories: $0,<5 \% ;+, 5$ $30 \%$;+, 31-50\%; +++, 51-75\%, and ++++, $>75 \%$. Counting was performed at x 200 magnification.

Positive and negative controls were performed to test the specific reaction of primary antibodies used in this study at a protein level. For positive control testing the sections and cells culture from synovium underwent an immunoperoxidase process. The positive immunolabeling for lubricin was cytoplasmic. For negative control testing sections and cells of cartilage were treated with normal rabbit serum instead of the specific antibodies.

\section{Western blot analysis}

The expression of lubricin was evaluated by Western blot analysis. The monolayer chondrocytes were washed twice with ice-cold PBS and collected with lysing buffer $(10 \mathrm{mM}$ Tris-HCl plus $10 \mathrm{mM} \mathrm{KCl}, 2 \mathrm{mM} \mathrm{MgCl}, 0.6 \mathrm{mM}$ PMSF, and $1 \%$ SDS, pH 7.4). After cooling for $30 \mathrm{~min}$ at $0^{\circ} \mathrm{C}$, cells were sonicated. Twenty micrograms of total protein, present in the supernatant, were loaded on each lane and separat-
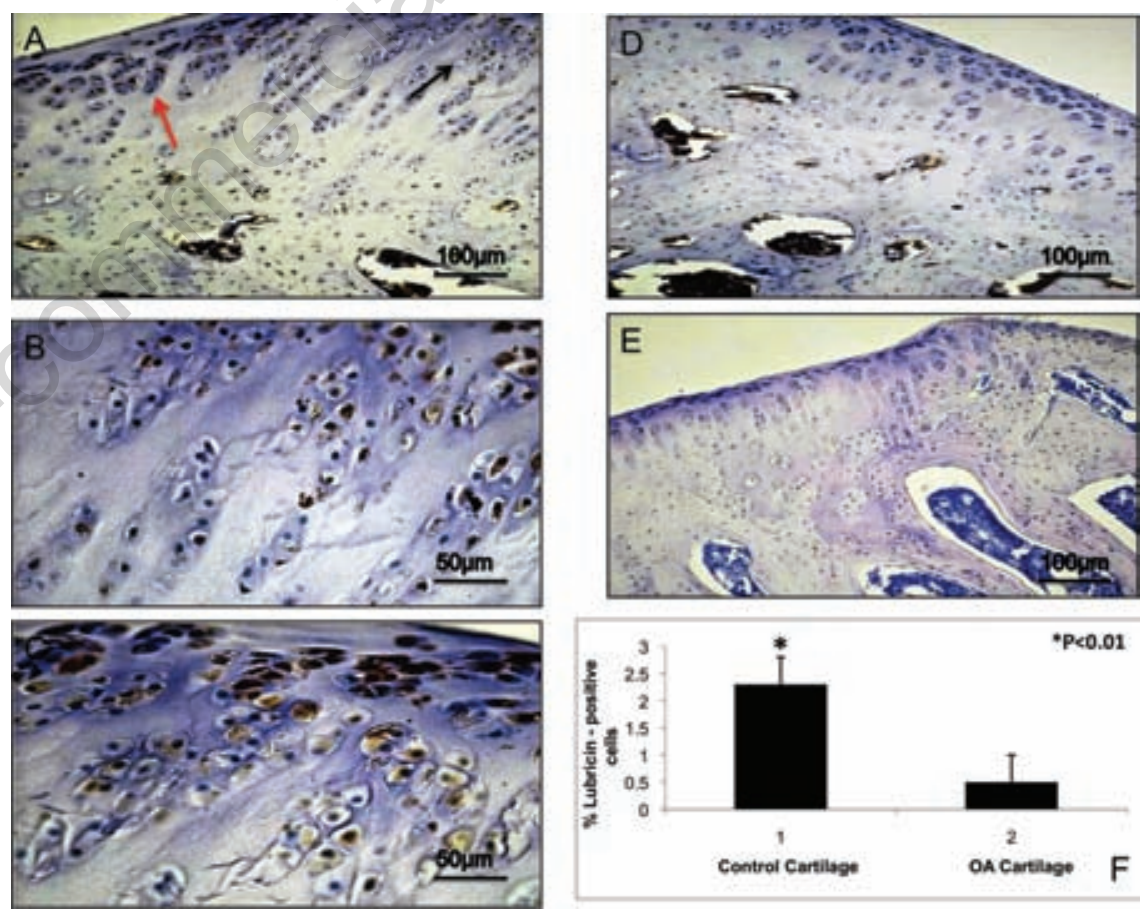

Figure 1. A) Lubricin immunohistochemistry (cytoplasmic and perinuclear immunolabeling) specimen from control cartilage (tissue explanted); strong lubricin immunolabeling of chondrocytes; magnification x20; scale bar: $100 \mu \mathrm{m}$. B) Magnification of panel A, black arrow; magnification x40; scale bar: $50 \mu \mathrm{m}$. C) magnification of panel A, red arrow; magnification x 40 ; scale bar: $50 \mu \mathrm{m}$. D) Lubricin immunohistochemistry specimen from OA cartilage (tissue explanted); weak lubricin immunolabeling of chondrocytes; magnification x20; scale bar: $100 \mu \mathrm{m}$. E) No immunoreaction was observed in the negative control treated with PBS without the primary antibodies (tissue explanted); magnification x20; scale bar: $100 \mu \mathrm{m}$. F) Percentage of lubricin positive cells out of the total number of cells counted in control cartilage and in OA cartilage. 


\section{Statistical analysis}

All variables were normally distributed. Comparisons between two means were tested with the Student's t-test; comparisons between different means were tested with ANOVA test. P-values of less than 0.01 were considered statistically significant. Ten fields from randomly selected slides were observed under fluorescence and a light microscope. Each field was photographed with a digital camera. On each photomicrograph three observers, blinded to the type of sample, identified and counted the number of total cells as well as the number of these cells exhibiting a positive reaction. The proportion of positive cells was calculated for each photomicrograph and a mean value was obtained for each sample. The results were expressed as a percentage. All data were analyzed with the SPSS program (SPSS $®$ release 16.0, Chicago, IL, USA).

Cohen's kappa was applied to measure the agreement between the three observers and averaged over all three to evaluate overall agreement using the following grading: 0-0.2 (slight), 0.21-0.40 (fair), 0.41-0.60 (moderate), 0.61-0.80 (substantial), and 0.81-1.0 (almost perfect).

\section{Results}

\section{Immunohistochemistry}

Lubricin immunohistochemical staining in explanted tissue was appreciated in an overall extracellular matrix (ECM) of cells of both normal and OA specimens. Articular cartilage displayed a layer of lubricin on the surface of tissues. However different patterns of immunopositive cells in the two sets of specimens were appreciated. Expression of lubricin was investigated via immunohistochemistry, in vivo explanted tissue. A very strong lubricin staining (IS, 4; ES, ++++) was observed in control cartilage where almost all chondrocytes were immunolabeled (Figure 1A,B,C). On the other hand a weak lubricin immunoreaction (IS, 1; $\mathrm{ES},+$ ) was appreciated in OA cartilage where few cells were immunostained (Figure 1D). No immunoreaction was observed in the negative control treated with PBS without the primary antibodies (Figure 1E). Both ES and IS were significantly greater $(\mathrm{P}<0.01)$ in control cartilage compared to $\mathrm{OA}$ cartilage. Interobserver agreement, measured using the Kappa coefficient, was 0.94 (almost perfect).

In PEGDA hydrogel light microscopy observation showed different patterns of immunopositive cells in the two sets of specimens. Expression of lubricin was investigated via immunohistochemistry, in control chondrocytes and in OA chondrocytes encapsulated in

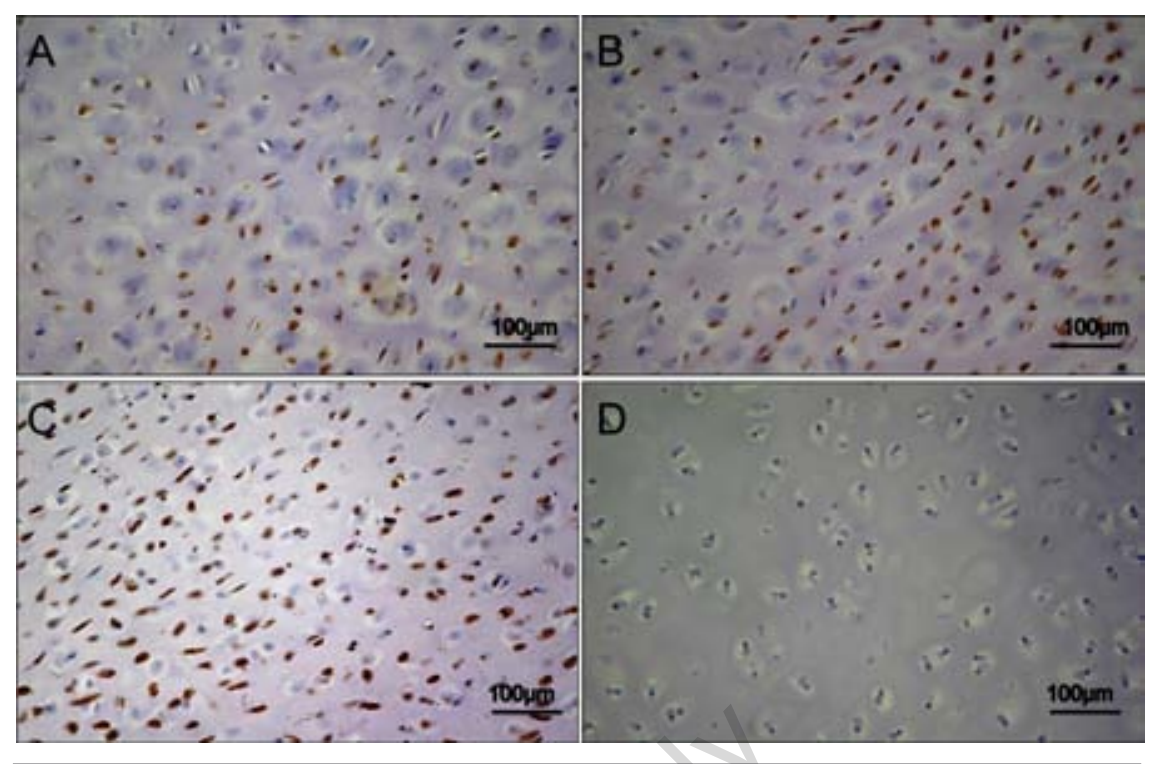

Figure 2. A) Lubricin immunohistochemistry (cytoplasmic and perinuclear immunolabeling) specimen from control chondrocytes after 3 weeks of encapsulations in PEGDA hydrogels. Strong lubricin immunolabeling in chondrocytes; magnification x20; scale bar: $100 \mu \mathrm{m}$. B) Lubricin immunostained specimen from control chondrocytes after 4 weeks of encapsulation in PEGDA hydrogels. Strong lubricin immunolabeling in chondrocytes; magnification x20; scale bar: $100 \mu \mathrm{m}$. C) Lubricin immunostained specimen from control chondrocytes after 5 weeks of encapsulations in PEGDA hydrogels; very strong lubricin immunolabeling in chondrocytes; magnification x20; scale bars: $100 \mu \mathrm{m}$. D) No immunoreaction was observed in the negative control treated with PBS without the primary antibodies; magnification x20; scale bars: $100 \mu \mathrm{m}$.

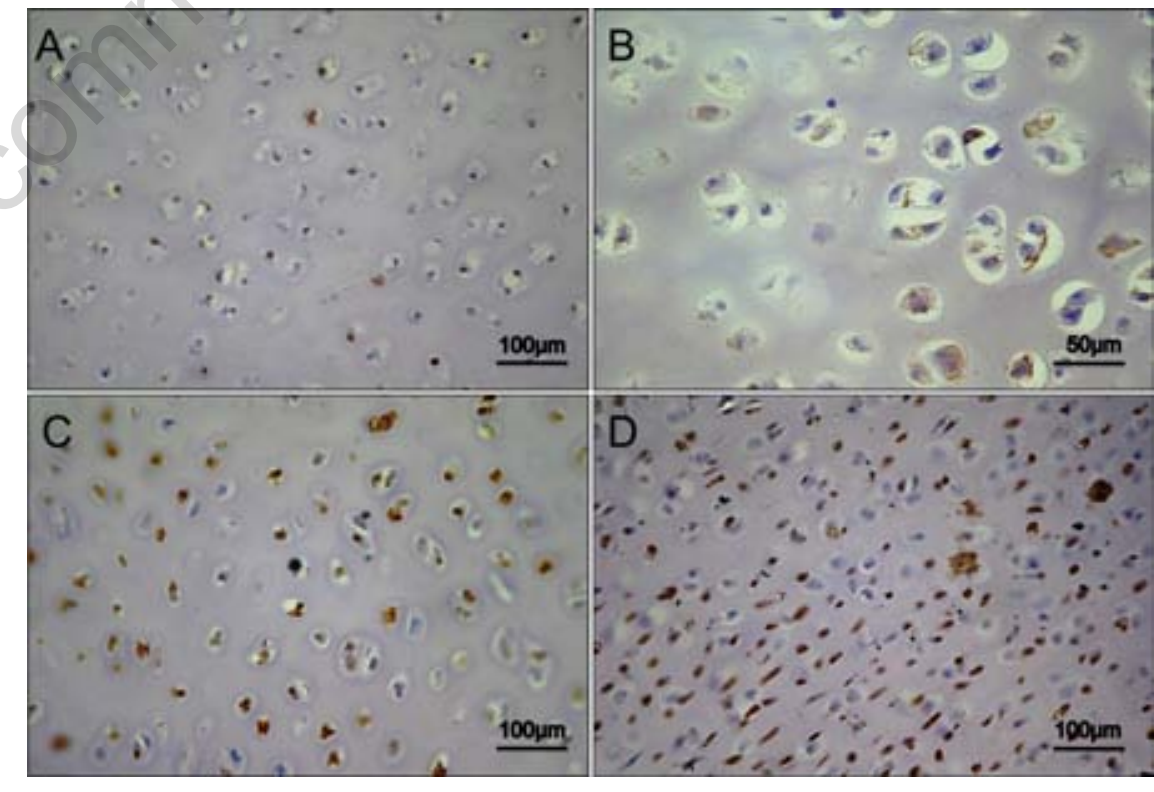

Figure 3. A) Lubricin immunohistochemistry specimen from OA chondrocytes after 3 weeks of encapsulations in PEGDA hydrogels; weak lubricin immunolabeling in chondrocytes; magnification x20; scale bars: $100 \mu \mathrm{m}$. B) Magnification $(x 40)$ of the panel A; scale bar: $50 \mu \mathrm{m}$. C) Lubricin immunostained specimen from OA chondrocytes after 4 weeks of encapsulation in PEGDA hydrogels; strong lubricin immunolabeling in the chondrocytes; magnification x20; scale bars: $100 \mu \mathrm{m}$. D) Lubricin immunostained specimen from $\mathrm{OA}$ chondrocytes after 5 weeks of encapsulation in PEGDA hydrogels; very strong lubricin immunolabeling in the chondrocytes; magnification x20; scale bar: $100 \mu \mathrm{m}$. 
PEGDA hydrogel at 3,4 and 5 weeks of culture. A strong (IS, 3; ES, +++) lubricin immunoreaction was shown in control chondrocytes after 3 weeks of culture and many chondrocytes were immunolabeled (Figure 2A). After 4 weeks of culture the immunoreactions was strong (IS, 3; ES, +++) (Figure 2B) and after 5 weeks of culture was very strong (Figure $2 \mathrm{C}$ ) (IS, 4 ; ES, ++++) were almost all the chondrocytes were immunolabeled by lubricin suggesting an increased expression. No immunoreaction was observed in the negative control (IS, 0 ; ES, 0) (Figure 2D). A weak/moderate (IS, 1; $\mathrm{ES},++)$ lubricin immunoreaction was seen in OA chondrocytes encapsulated in PEGDA hydrogels after the 3rd week of culture and nearly half chondrocytes were immunostained (Figure 3A, B). After 4 weeks of culture strong (IS, 3 ; ES, +++) lubricin immunoreaction was demonstrated in $\mathrm{OA}$ chondrocytes encapsulated in PEGDA hydrogels (Figure 3C) and after 5 weeks of culture was very strong compared with control chondrocytes (IS, 4; ES, ++++) (Figure 3D). IS and ES were significantly greater $(\mathrm{P}<0.01)$ in control chondrocytes compared with $0 A$ chondrocytes, at the $3^{\text {rd }}, 4^{\text {th }}$ and $5^{\text {th }}$ weeks (Figure 4). Interobserver agreement, measured using the Kappa coefficient, was 0.91 (almost perfect).

\section{Immunocytochemistry}

Fluorescence microscopy observation showed a detection of lubricin in cells from control cartilage and in 0A cartilage after the 4 passages using immunocytochemistry. Strong (IS, 4; ES, ++++) lubricin immunoreaction was observed in control cartilage (Figure 5A). The chondrocytes from OA cartilage exhibited a weak (IS, 1; ES, +) lubricin immunoreaction, with only few cells fluorescing (Figure 5B). No immunoreaction was observed in the negative control treated with $1 \%$ BSA without the primary antibodies (Figure 5C). ES was significantly greater $(\mathrm{P}<0.01)$ in control chondrocytes compared to $\mathrm{OA}$ chondrocytes (Figure 5D). Interobserver agreement, measured using the Kappa coefficient, was 0.91 (almost perfect).

\section{Western blot}

In this study, we also examined, by Western blot, the production of lubricin in human monolayer chondrocytes from normal and $\mathrm{OA}$ cartilage. On the $24^{\text {th }}$ day we have investigated the effects of different cultures on the release of lubricin. Negligible amounts of lubricin were produced by monolayer chondrocytes from $0 A$ cartilage. In contrast, lubricin potently was induced by chondrocytes from normal cartilage (Figure 6).

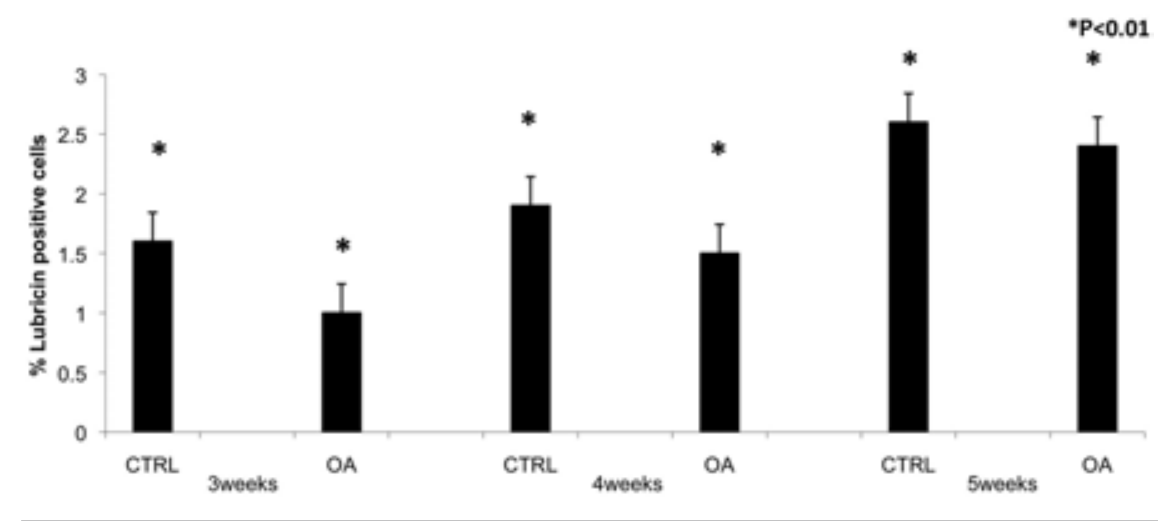

Figure 4. Percentage of lubricin positive cells out of the total number of cells counted in control chondrocytes and in OA chondrocytes after 3, 4 and 5 weeks in PEGDA hydrogels.
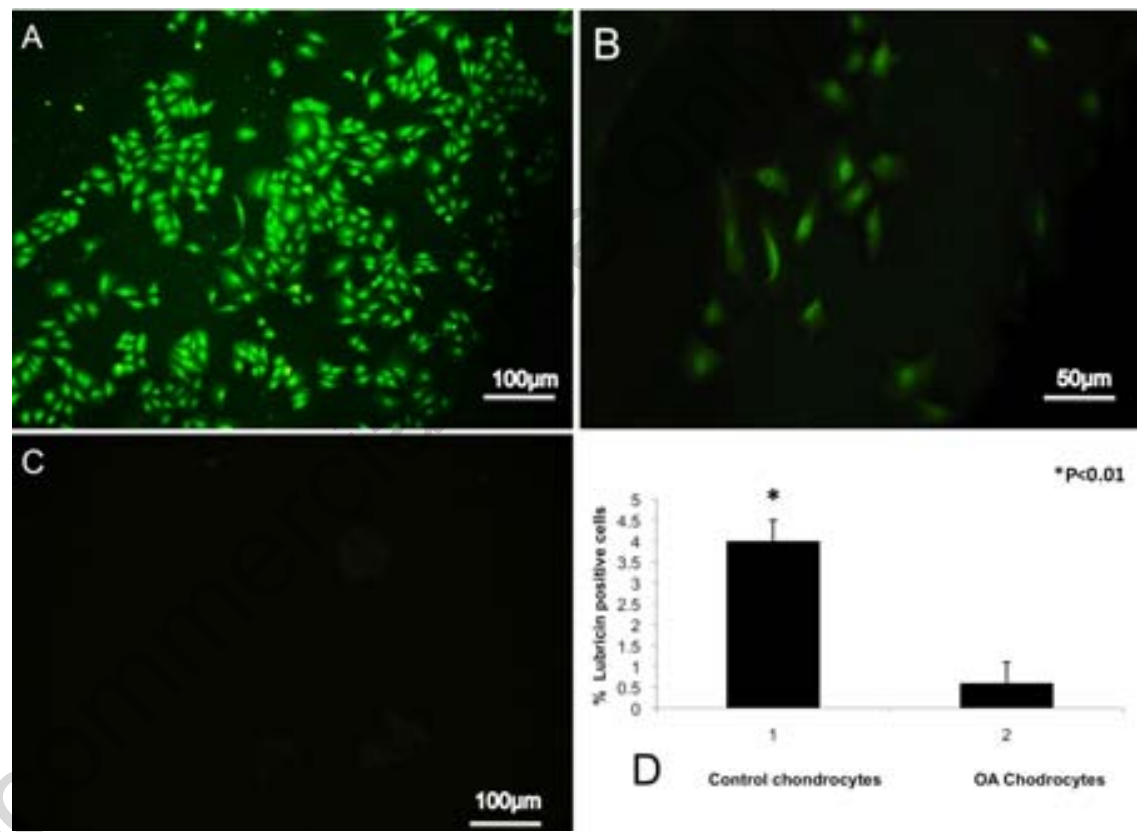

Figure 5. A) Lubricin immunocytochemistry (cytoplasmic and perinuclear immunolabeling) specimen from control chondrocytes; strong lubricin immunolabeling in cells at fourth passage of culture; magnification x20; scale bars: $100 \mu \mathrm{m}$. B) Lubricin immunocytochemistry (cytoplasmic and perinuclear immunolabeling) specimen from $\mathrm{OA}$ chondrocytes; weak lubricin immunolabeling in cells at fourth passage of culture; magnification x20; scale bar: $50 \mu \mathrm{m}$. C) No immunoreaction was observed in the negative control treated with PBS without the primary antibodies; magnification x20; scale bar: $100 \mu \mathrm{m}$. D) Percentage of lubricin positive cells out of the total number of cells counted in control chondrocytes and in OA chondrocytes.

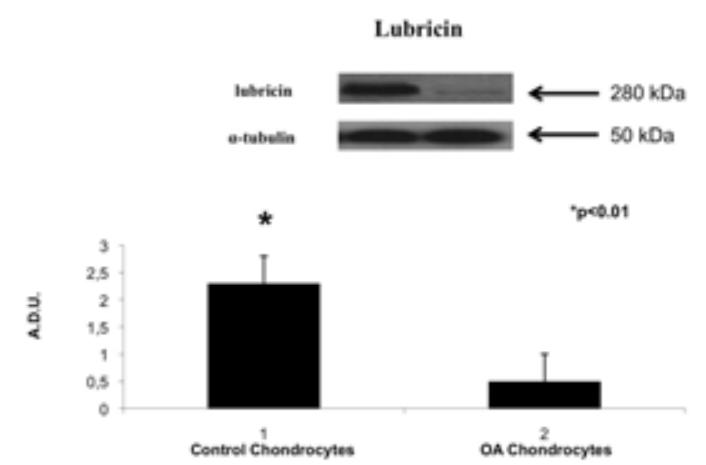

Figure 6. Lubricin expression induced in human monolayer chondrocytes from normal cartilage and human monolayer chondrocytes from OA cartilage determined by Western blot analysis. Data show the relative expression (mean \pm SEM) of lubricin calculated as arbitrary densitometric units (A.D.U.) collected from three independent experiments. ${ }^{*} \mathbf{P}<\mathbf{0 . 0 1}$ compared to monolayer chondrocytes. 


\section{Discussion}

New therapeutic strategies for OA cartilage deterioration are a novel biomedical challenge. As we know, tissue engineering focuses on restoration, maintenance, or improvement of tissue function through the development of biological substitutes. The principle obstacle for clinical transplantation today is the shortage of donor organs. For this reason, tissue engineering and regenerative medicine have become promising and important fields of research that may offer new sources of tissues and organs for transplantation. Poly (ethylene glycol) (PEG) based hydrogels have attracted wide interest as a scaffolding material for tissue engineering applications. ${ }^{29-32}$ Hydrogels encompass a class of scaffolds with many attractive features for cartilage tissue engineering. ${ }^{29-32}$ Hydrogels are made from watersoluble polymer chains that are cross-linked to form water-insoluble gel networks. Cells in the polymer solution can be encapsulated into the hydrogel during the cross-linking process. The resulting gel network can be designed to have a high water content, enabling efficient transport of nutrient and waste products to and from cells. Polymer cross-linking and hydrogel formation can be achieved by various physical and chemical mechanisms. ${ }^{33}$ This scaffold, like some others, is thus designed to perform a number of biological functions including stimulation of cells to synthesize ECM, proliferate, migrate, prevent apoptosis, and/or differentiate. The characterization of extracellular matrix anabolic factors and proteins produced by cells in the scaffolds is critical to evaluate the success of a tissue engineering system.

In this study we have evaluated lubricin expression in chondrocytes from articular cartilage of the knee, from patients with $\mathrm{OA}$ and compared them to normal cartilage chondrocytes, both in vivo, on paraffin sections, this evaluation was also carried out after chondrocytes encapsulation in hydrogel PEGDA, in vitro after isolation of these chondrocytes and through western blot analysis.

Lubricin is a chondroprotective glycoprotein, which acts as a vital counteragent against aberrant protein and/or cellular adhesion, infiltration and over-proliferation, and serves as a critical boundary lubricant between opposing cartilage surfaces. This protein is less expressed with aging and during $\mathrm{OA}$, moreover the lubricin gene is differently expressed in the synovium of RA and OA implying a possible role in the pathogenesis of these diseases. ${ }^{34}$ Furthermore, impaired lubricin expression is associated with the onset of early $\mathrm{OA}$ in a sheep meniscectomy model. ${ }^{35}$

Here, for the first time, we have shown that lubricin is down regulated in $\mathrm{OA}$ cartilage explants and cell cultures from knee joints. This result is in line with previous studies that advocate intraarticular supplementation with lubricin for $\mathrm{OA}$ joints. ${ }^{36,37}$ More interestingly, we demonstrated in our study that OA chondrocytes encapsulated in PEGDA scaffolding are able to restore lubricin biosynthesis. In fact after 4 weeks of culture of $0 \mathrm{~A}$ chondrocytes encapsulated in PEGDA hydrogel, lubricin expression nearly reached the level of the control scaffold. This increased expression of lubricin in $\mathrm{OA}$ chondrocytes may, somehow, be related to the absence of cytokines, which seem to influence lubricin biosynthesis. A disregulation of lubricin metabolism, under the influence of elevated cytokine concentrations in diseased or damaged joints, has been described, ${ }^{14,38-40}$ and this, in time, might thereby lead to lubrication deficiencies and loss of function. In this respect, it has been claimed that a decrease in synovial fluid lubricin concentrations following anterior cruciate ligament injury may place the joint at an increased risk of wear-induced damage, as a consequence of lack of boundary lubrication, potentially leading to secondary osteoarthritis.

In this study, we clearly demonstrated that OA chondrocytes grown in PEGDA hydrogels were able to express lubricin. This investigation shows an increased expression of lubricin in chondrocytes encapsulated in hydrogel PEGDA scaffolding from OA patients after 3,4 and 5 weeks of culture. In particular, chondrocytes from OA cartilage after 5 weeks of culture in hydrogels (PEGDA) showed an increased expression of lubricin comparable to the control cartilage. These are preliminary data, and further studies using a longer incubation time will be useful to better understand the potential effect of PEGDA. In conclusion our study suggests the possibility of applying autologous cell transplantation (ACT) in conjunction with scaffolding materials for repairing cartilage lesions in patients with $\mathrm{OA}$ in order to reduce, at least, the progression of the disease.

\section{References}

1. Loreto C, Musumeci G, Leonardi R. Chondrocyte-like apoptosis in temporomandibular joint disc internal derangement as a repair-limiting mechanism. An in vivo study. Histol Histopathol 2009;24: 293-8.

2. Musumeci G, Loreto C, Carnazza ML, Martinez G. Characterization of apoptosis in articular cartilage derived from the knee joints of patients with osteoarthritis. Knee Surg Sports Traumatol Arthrosc 2011;19:307-13.

3. Schulze-Tanzil G. Activation and dediffer- entiation of chondrocytes: implications in cartilage injury and repair. Ann Anat 2009; 191:325-38.

4. Burrage PS, Mix KS, Brinckerhoff CE. Matrix metalloproteinases: role in arthritis. Front Biosci 2006;11:529-43.

5. Pritzker KP, Gay S, Jimenez SA, Ostergaard K, Pelletier JP, Revell PA, et al. Osteoarthritis cartilage histopathology: grading and staging. Osteoarthritis Cartilage 2006;14:13-29.

6. Lorenz H, Richter W. Osteoarthritis: cellular and molecular changes in degenerating cartilage. Prog Histochem Cytochem 2006;40:135-63.

7. Buckwalter JA, Mankin HJ. Articular cartilage: degeneration and osteoarthritis, repair, regeneration, and transplantation. Instr Course Lect 1998;47:487-504.

8. Goldring SR, Goldring MB. The role of cytokines in cartilage matrix degeneration in osteoarthritis. Clin Orthop Relat Res 2004:S27-36.

9. Aigner T, Soeder S, Haag J. IL-1beta and BMPs--interactive players of cartilage matrix degradation and regeneration. Eur Cell Mater 2006;12:49-56.

10. Murray TG, Parker RD. Restoring cartilage defects: microfracture to autologous chondrocyte implantation using investigational 3D scaffold. Orthopedics 2007;30:766-67.

11. Petersen W, Zelle S, Zantop T. Arthroscopic implantation of a three dimensional scaffold for autologous chondrocyte transplantation. Arch Orthop Trauma Surg 2008;128: 505-8.

12. Lee CR, Grodzinsky AJ, Hsu HP, Spector M. Effects of a cultured autologous chondrocyte-seeded type II collagen scaffold on the healing of a chondral defect in a canine model. J Orthop Res 2003;21:272-81.

13. Grad S, Lee CR, Wimmer MA, Alini M. Chondrocyte gene expression under applied surface motion. Biorheology 2006; 43:259-69.

14. Jones AR, Flannery CR. Bioregulation of lubricin expression by growth factors and cytokines. Eur Cell Mater 2007; 13:40-5.

15. Zhang D, Johnson LJ, Hsu HP, Spector M. Cartilaginous deposits in subchondral bone in regions of exposed bone in osteoarthritis of the human knee: histomorphometric study of PRG4 distribution in osteoarthritic cartilage. J Orthop Res 2007;25:873-83.

16. Elsaid KA, Jay GD, Chichester CO. Reduced expression and proteolytic susceptibility of lubricin/superficial zone protein may explain early elevation in the coefficient of friction in the joints of rats with antigen-induced arthritis. Arthritis Rheum 2007;56:108-16.

17. Rhee DK, Marcelino J, Baker M, Gong Y, 
Smits P, Lefebvre V, et al. The secreted glycoprotein lubricin protects cartilage surfaces and inhibits synovial cell overgrowth. J Clin Invest 2005;115:622-31.

18. Funakoshi T, Spector M. Chondrogenic differentiation and lubricin expression of caprine infraspinatus tendon cells. J Orthop Res 2010;28:716-25.

19. Taguchi M, Zhao C, Sun YL, Jay GD, An KN, Amadio PC. The effect of surface treatment using hyaluronic acid and lubricin on the gliding resistance of human extrasynovial tendons in vitro. $\mathrm{J}$ Hand Surg Am 2009;34:1276-81.

20. Taguchi M, Sun YL, Zhao C, Zobitz ME, Cha CJ, Jay GD, et al. Lubricin surface modification improves tendon gliding after tendon repair in a canine model in vitro. J Orthop Res 2009;27:257-63.

21. Sun Y, Chen MY, Zhao C, An KN, Amadio PC. The effect of hyaluronidase, phospholipase, lipid solvent and trypsin on the lubrication of canine flexor digitorum profundus tendon. J Orthop Res 2008;26:12259.

22. Funakoshi T, Schmid T, Hsu HP, Spector M. Lubricin distribution in the goat infraspinatus tendon: a basis for interfascicular lubrication. J Bone Joint Surg Am 2008; 90:803-14.

23. Taguchi M, Sun YL, Zhao C, Zobitz ME, Cha CJ, Jay GD, et al. Lubricin surface modification improves extrasynovial tendon gliding in a canine model in vitro. $\mathrm{J}$ Bone Joint Surg Am 2008;90:129-35.

24. Sun Y, Berger EJ, Zhao C, Jay GD, An KN, Amadio PC. Expression and mapping of lubricin in canine flexor tendon. J Orthop Res 2006;24:1861-8.

25. Klein SM, Steele SM, Nielsen KC, Pietrobon R, Warner DS, Martin A, et al. The difficulties of ambulatory interscalene and intra-articular infusions for rotator cuff surgery: a preliminary report. Can J Anaesth 2003;50:265-9.

26. Schmidt TA, Schumacher BL, Klein TJ, Voegtline MS, Sah RL. Synthesis of proteoglycan 4 by chondrocyte subpopulations in cartilage explants, monolayer cultures, and resurfaced cartilage cultures. Arthritis Rheum 2004;50:2849-57.

27. van der Sluijs JA, Geesink RG, van der Linden AJ, et al. The reliability of the Mankin score for osteoarthritis. J Orthop Res 1992;10:58-61.

28. Petersson IF, Boegård T, Saxne T, et al. Radiographic osteoarthritis of the knee classified by the Ahlbäck and Kellgren \& Lawrence systems for the tibiofemoral joint in people aged 35-54 years with chronic knee pain. Ann Rheum Dis 1997;56:493-6.

29. Kim J, Hefferan TE, Yaszemski MJ, Lu L. Potential of hydrogels based on poly(ethylene glycol) and sebacic acid as orthopedic tissue engineering scaffolds. Tissue Eng Part A 2009;15:2299-307.

30. Akdemir ZS, Akcakaya H, Kahraman MV, Ceyhan T, Kayaman-Apohan N, Gungor A. Photopolymerized injectable RGD-modified fumarated poly(ethylene glycol) diglycidyl ether hydrogels for cell growth. Macromol Biosci 2008;8:852-62.

31. Xin AX, Gaydos C, Mao JJ. In vitro degradation behavior of photopolymerized PEG hydrogels as tissue engineering scaffold. Conf Proc IEEE Eng Med Biol Soc 2006;1:2091-3.

32. Ji Y, Ghosh K, Shu XZ, Li B, Sokolov JC, Prestwich GD, et al. Electrospun threedimensional hyaluronic acid nanofibrous scaffolds. Biomaterials 2006;27:3782-92.

33. Gutowska A, Jeong B, Jasionowski M. Injectable gels for tissue engineering. Anat Rec 2001;263:342-9.

34. Justen HP, Grunewald E, Totzke G, Gouni-
Berthold I, Sachinidis A, Wessinghage D, et al. Differential gene expression in synovium of rheumatoid arthritis and osteoarthritis. Mol Cell Biol Res Commun 2000; 3:165-72.

35. Young AA, McLennan S, Smith MM, Smith SM, Cake MA, Read RA, et al. Proteoglycan 4 downregulation in a sheep meniscectomy model of early osteoarthritis. Arthritis Res Ther 2006;8:R41.

36. Flannery CR, Zollner R, Corcoran C, Jones AR, Root A, Rivera-Bermudez MA, et al. Prevention of cartilage degeneration in a rat model of osteoarthritis by intraarticular treatment with recombinant lubricin. Arthritis Rheum 2009;60:840-7.

37. Lee SY, Nakagawa T, Reddi AH. Induction of chondrogenesis and expression of superficial zone protein (SZP)/lubricin by mesenchymal progenitors in the infrapatellar fat pad of the knee joint treated with TGF-betal and BMP-7. Biochem Biophys Res Commun 2008;376:148-53.

38. DuRaine G, Neu CP, Chan SM, Komvopoulos K, June RK, Reddi AH. Regulation of the friction coefficient of articular cartilage by TGF-betal and IL-1beta. J Orthop Res 2009;27:249-56.

39. Elsaid KA, Fleming BC, Oksendahl HL, Machan JT, Fadale PD, Hulstyn MJ, et al. Decreased lubricin concentrations and markers of joint inflammation in the synovial fluid of patients with anterior cruciate ligament injury. Arthritis Rheum 2008;58:1707-15.

40. Schmidt TA, Gastelum NS, Han EH, Nugent-Derfus GE, Schumacher BL, Sah RL. Differential regulation of proteoglycan 4 metabolism in cartilage by IL-1alpha, IGF-I, and TGF-betal. Osteoarthritis Cartilage 2008;16:90-7. 